\title{
Association of lipid peroxidation and antioxidant status with metabolic syndrome in Iranian healthy elderly women
}

\author{
AFSANEH BAKHTIARI $^{1}$, KARIMOLLA HAJIAN-TILAKI ${ }^{2}$, \\ SHABNAM OMIDVAR $^{1}$ and FATEMEH NASIRI AMIRI ${ }^{1,3}$
}

\begin{abstract}
Departments of ${ }^{1}$ Midwifery and ${ }^{2}$ Biostatistics and Epidemiology, Faculty of Medicine, Babol University of Medical Sciences; ${ }^{3}$ Infertility and Health Reproductive Research Center, Health Research Institute, Babol University of Medical Sciences, Babol 47176-47745, Iran
\end{abstract}

Received July 17, 2017; Accepted July 27, 2017

DOI: $10.3892 /$ br.2017.964

\begin{abstract}
The interconnection between aging and metabolic syndrome (MetS) and their effect on oxidative stress $(\mathrm{OxS})$ status lacks adequate information. Additionally, the agerelated changes of antioxidant defenses and $\mathrm{OxS}$ in senior women with MetS in comparison to healthy senior women are not yet established. We analyzed the correlation between oxidative defense status and $\mathrm{OxS}$ with MetS components. Through further examination of MetS and aging, we aimed to determine their independent effects on $\mathrm{OxS}$ and oxidative defense status. This community-based cross-sectional study was conducted in the rural area of Babol, Iran. A total of 75 women of $\geq 60$ years of age with MetS along with 89 women with similar conditions without the MetS, serving as the control group, were studied. Blood glucose, lipid profile, malondialdehyde (MDA) and total antioxidant capacity (TAC) were determined. Data were analyzed using multiple linear regression, ANOVA and independent t-tests. MDA and TAC levels independently showed a significant correlation with triglyceride (TG), waist circumference, fasting blood glucose and high-density lipoprotein cholesterol (HDL-C). As suggested by the standardized B (0.810, -0.783 , $\mathrm{P}<0.001 ;-0.052, \mathrm{P}<0.001,0.047, \mathrm{P}<0.01)$, TG followed by HDL-C were the most strongly correlated factors with MDA and TAC. Furthermore, MetS and age were independent risk factors for antioxidant activity reduction and OxS. However, MetS had a much higher predictive power than age (standardized B 0.573 for MetS and 0.376 for age, $\mathrm{P}<0.001$ ). Aging and MetS, both lead to OxS, but the impact of MetS on this disorder was far greater than the effect of age. However, their cumulative effects can lead to a worsening of the situation.
\end{abstract}

Correspondence to: Dr Fatemeh Nasiri Amiri, Department of Midwifery, Faculty of Medicine, Babol University of Medical Sciences, Ganjafrouz Street, Babol 47176-47745, Iran

E-mail: nasiri_fa@yahoo.com

Key words: oxidative stress, lipid peroxidation, total antioxidant, capacity, metabolic syndrome, aging, elderly women
Therefore, early diagnosis and treatment of MetS, especially in the elderly can prevent any adverse impact of MetS.

\section{Introduction}

Oxidative stress $(\mathrm{OxS})$ is a well-known factor that plays a key role in cardiovascular diseases (CVD). It is the result of an imbalance between the prooxidants and antioxidants in the body (1). Malondialdehyde (MDA) is the product of OxS and lipid peroxidation (2) and total antioxidant capacity (TAC) assays have been designed to determine overall antioxidant power. Measuring of the two markers in blood serves as a selective method for monitoring $\mathrm{OxS}$ and oxidative defense status in humans, respectively (3).

Evidence suggests that $\mathrm{OxS}$ is increased in the case of metabolic syndrome (MetS) due to fat accumulation (4). Dyslipidemia and insulin resistance (IR) associated with MetS increases the production of reactive oxygen species and consequently raises the oxidation of lipid products, DNA and proteins, which in turn lead to endothelium dysfunction, cancer and other chronic diseases related to aging $(5,6)$. On the other hand, aging is accompanied by an increase in oxidative damage due to an impaired physiological function (6). Regardless of age, menopause contributes to the development of MetS on the direct effects of sex hormones. Some features of MetS (e.g., diabetes and hypertension) create a greater risk for CVD in women (7). It seems that the transition from premenopausal to postmenopausal period is associated with metabolic fundamental changes. In many women, the features of MetS (abdominal adiposity, IR and dyslipidemia) emerge with estrogen deficiency. Postmenopausal status is associated with a $60 \%$ increased risk of MetS, even after adjusting for the confounding variables (1).

There are a relatively large number of studies looking at changes in TAC and MDA with certain metabolic conditions such as diseases, inactivity and obesity $(8,9)$, but the results of studies investigating $\mathrm{OxS}$ in aging are controversial. In institutionalized or frail subjects, $\mathrm{OxS}$ was reported to increase which was related to low antioxidant status, while in indepently living elderly this increase is not always significant (10). The age-related alterations of antioxidant defenses and $\mathrm{OxS}$ in elderly women with MetS compared with healthy 
elderly individuals are not yet ascertained and there is a lack of information regarding the interactions between MetS and aging on $\mathrm{OxS}$ status.

Due to the aging process in Iran and the increased prevalence of MetS (11-13), we examined the association between the OxS and oxidative defense status with the MetS components. In addition, we aimed to establish the independent effect of MetS and aging on the status of OxS and oxidative defense status.

\section{Materials and methods}

Patient selection and study design. This community-based cross-sectional study was conducted on 164 women of $\geq 60$ years of age who resided in rural areas of the central part of Babol, Iran. Each village has a Health Center affiliated with the Babol University of Medical Sciences (Babol, Iran). The participants were recruited through community advertisement by poster. The posters were distributed in the rural health centers. The poster included information regarding the objectives of the study, the eligibility criteria and the study procedures. One cohort of 306 volunteers was initially assessed for eligibility on a consecutive basis. A total of 142 volunteers were excluded: 46 , for not meeting the primary criteria; 76, had one or more of the exclusion criteria; and 20, were not interested. The exclusion criteria were any current or previous use of estrogen therapy and antioxidant medicines, history of CVD, hyper- and hypothyroidism, kidney, liver diseases, cancer and smoking. Therefore, a total number of 164 eligible women, aged $\geq 60$ years were included and the data were analyzed. The MetS was measured with regards to the ATP III guideline as follows: waist circumference (WC) $>80 \mathrm{~cm}$; serum high-density lipoprotein cholesterol (HDL-C) $<50 \mathrm{mg} / \mathrm{dl}$; triglyceride $(\mathrm{TG}) \geq 150 \mathrm{mg} / \mathrm{dl}$; fasting blood glucose $(\mathrm{FBG}) \geq 100 \mathrm{mg} / \mathrm{dl}$; and systolic blood pressure $\geq 130 \mathrm{mmHg}$ and diastolic $\geq 85 \mathrm{mmHg}$. To qualify for the MetS, the participants were required to have a minimum of three criteria without taking any medicines for hyperlipidemia, hypertension and diabetes (14).

Each participant was interviewed with a structured questionnaire to collect demographic information. There was no smoker among the samples and all were homogeneous in terms of age, disease conditions and lifestyle. A written and signed informed consent form was completed by all the participants. The study was approved by the Medical Research Ethics Committee of Babol University of Medical Sciences.

Measurements. After 10-12 h of overnight fasting, a blood sample was taken. After clot formation, the clots were gently separated from the tubes by use of a wooden applicator and then the blood samples were centrifuged for $10 \mathrm{~min}$ at $1,000 \mathrm{x} g$ to obtain the serum samples.

The total cholesterol (TC) and TG levels were measured using Elitech kit (ELITech Group, Puteaux, France) and HDL-C, low-density lipoprotein cholesterol (LDL-C) and LDL-C (VLDL-C), all from Pars Azmoon kit (Pars Azmoon Co., Tehran, Iran). FBG was also measured on the day of blood collection by the Pars Azmoon kit. Lipid profiles and FBG were assayed on Autoanalyzer (Mindray-BS 300; Mindray, Shenzhen, China). MDA measurements were evaluated by
Table I. Clinical characteristics of older women with and without metabolic syndrome $(n=164)$.

\begin{tabular}{lcc}
\hline Variables & $\begin{array}{c}\text { MetS }(-) \\
(\mathrm{n}=89)\end{array}$ & $\begin{array}{c}\text { MetS }(+) \\
(\mathrm{n}=75)\end{array}$ \\
\hline Age (years) & $64.6 \pm 2.9$ & $64.2 \pm 2.8$ \\
Age of menopause (years) & $47.7 \pm 4.7$ & $48.2 \pm 4.08$ \\
BMI $\left(\mathrm{kg} / \mathrm{m}^{2}\right)$ & $26.6 \pm 0.60$ & $27.3 \pm 0.72$ \\
WC $(\mathrm{cm})$ & $89.6 \pm 5.82$ & $92.3 \pm 7.45$ \\
TC $(\mathrm{mg} / \mathrm{dl})$ & $215.2 \pm 28$ & $228.9 \pm 24^{\mathrm{a}}$ \\
HDL-C $(\mathrm{mg} / \mathrm{dl})$ & $61.1 \pm 5.34$ & $46.8 \pm 3.80^{\mathrm{b}}$ \\
Non-HDL-C $(\mathrm{mg} / \mathrm{dl})$ & $162.1 \pm 7.23$ & $187 \pm 5.20^{\mathrm{b}}$ \\
TG $(\mathrm{mg} / \mathrm{dl})$ & $157.2 \pm 30.50$ & $204.3 \pm 23.25^{\mathrm{b}}$ \\
FBG $(\mathrm{mg} / \mathrm{dl})$ & $94.3 \pm 2.12$ & $103.4 \pm 2.25^{\mathrm{b}}$ \\
SBP $(\mathrm{mmHg})$ & $132.3 \pm 0.41$ & $137 \pm 0.50$ \\
DBP $(\mathrm{mmHg})$ & $73.6 \pm 1.15$ & $73.4 \pm 1.33$ \\
MDA $(\mu \mathrm{mol} / \mathrm{l})$ & $3.6 \pm 0.17$ & $5.1 \pm 0.28^{\mathrm{b}}$ \\
TAC $(\mathrm{mmol} / \mathrm{l})$ & $1.505 \pm 0.0453$ & $1.304 \pm 0.045^{\mathrm{b}}$ \\
& &
\end{tabular}

Values are mean \pm standard deviation. BMI, body mass index; WC, waist circumference; TC, total cholesterol; HDL-C, high-density lipoprotein cholesterol; TG, triglyceride; FBG, fasting blood glucose; SBP, systolic blood pressure; DBP, diastolic blood pressure; MDA, malondialdehyde; TAC, total antioxidant capacity. Non HDL-C was calculated by the Fried-Wald formula (TC-HDL-C). ${ }^{a} \mathrm{P}<0.01$, ${ }^{\mathrm{b}} \mathrm{P}<0.001$.

the thiobarbituric acid reactive substances (TBARS) method based on the method described by Ruiz-Ramos et al (15). In this assay, the MDA was conjugated with thiobarbituric acid (Merck KGaA, Darmstadt, Germany), and the absorbance was read against blank at $535 \mathrm{~nm}$. OxS was defined as MDA $\geq 5 \mu \mathrm{mol} / 1$ (16). The TAC level was determined by use of the ferric-reducing antioxidant power (FRAP) technique. Based on Benzie and Strain (17), the assay was performed using tri-pyridyl-s-triazine reagent. This technique is used to calculate sample antioxidant reducing strength to convert ferric-tripyridyltriazine to a ferrous form with the light absorbance at $593 \mathrm{~nm}$. The FRAP level was measured by plotting a standard curve of absorbance against standard solution Fe (II) concentration (mmol/l). Blood pressure was assessed on the right arm by a calibrated mercury sphygmomanometer. The WC was determined at the mid-point between the iliac crest and the lowest rib and hip at the widest point.

Statistical analysis. Clinical characteristics of the participants in the two groups (with and without MetS) were evaluated by independent t-test. Multiple linear regression analysis was performed with MetS components as the independent variables and MDA and TAC levels as the dependent variables. Moreover, multiple linear regression analysis with MetS (no, yes) and age $\geq 65$ (no, yes) as the dichotomous independent variable was conducted to determine the independent effect of MetS and aging on the status of $\mathrm{OxS}$ and antioxidant defense. The resulting unstandardized coefficients B (standard error) and standardized coefficients $\beta$ were reported. 
Table II. The regression coefficient of metabolic syndrome components on MDA and TAC levels in elderly women (n=164).

\begin{tabular}{|c|c|c|c|c|c|c|}
\hline \multirow[b]{2}{*}{ Parameters } & \multicolumn{3}{|c|}{ MDA } & \multicolumn{3}{|c|}{ TAC } \\
\hline & $\mathrm{B}(\mathrm{SE})$ & $\beta$ & P-value & $\mathrm{B}(\mathrm{SE})$ & $\beta$ & P-value \\
\hline WC & $0.522(0.29)$ & 0.431 & $<0.01$ & $-0.037(0.007)$ & -0.231 & $<0.01$ \\
\hline SBP & $0.251(0.038)$ & 0.079 & 0.842 & $-0.006(0.003)$ & -0.072 & 0.678 \\
\hline DBP & $0.246(0.05)$ & 0.064 & 0.674 & $0.005(0.001)$ & 0.046 & 0.735 \\
\hline TG & $0.810(0.03)$ & 0.464 & $<0.001$ & $-0.052(0.001)$ & -0.577 & $<0.001$ \\
\hline HDL-C & $-0.783(0.02)$ & -0.450 & $<0.001$ & $0.047(0.004)$ & 0.336 & $<0.01$ \\
\hline FBG & $0.642(0.034)$ & 0.396 & $<0.01$ & $-0.042(0.002)$ & -0.312 & $<0.01$ \\
\hline
\end{tabular}

$\mathrm{P}<0.05$ multiple regression analysis, significant differences; $\mathrm{B}$, standardized regression coefficient; $\beta$, unstandardized regression coefficient; WC, waist circumference; SBP, systolic blood pressure; DBP, diastolic blood pressure; TG, triglyceride; HDL-C, high-density lipoprotein cholesterol; FBG, fasting blood glucose; MDA, malondialdehyde; TAC, total antioxidant capacity.

Table III. Number of MetS components and MDA and TAC in elderly women $(n=164)$.

\begin{tabular}{lcc}
\hline $\begin{array}{l}\text { No. of MetS } \\
\text { components }\end{array}$ & $\begin{array}{c}\text { MDA } \\
\text { Mean } \pm \text { SD }\end{array}$ & $\begin{array}{c}\text { TAC } \\
\text { Mean } \pm \text { SD }\end{array}$ \\
\hline $0-1$ & $2.67 \pm 0.54$ & $1.303 \pm 0.015$ \\
2 & $3.04 \pm 0.48$ & $1.182 \pm 0.018$ \\
3 & $4.12 \pm 0.36$ & $1.069 \pm 0.012$ \\
$\geq 4$ & $5.31 \pm 0.62$ & $0.882 \pm 0.017$ \\
P-value & $\mathrm{P}<0.001$ & $\mathrm{P}<0.001$ \\
\hline
\end{tabular}

MetS, metabolic syndrome; MDA, malondialdehyde; TAC, total antioxidant capacity; SD, standard deviation

In addition, the participants were divided into 4 groups according to the quartiles of MDA and TAC; variables were compared by ANOVA test among these quartiles, respectively. The ANOVA test was also applied to compare the mean MDA and TAC according to the number of MetS components. The statistical analyses were all performed by using IBM SPSS (Armonk, NY, USA) for windows (version 23). $\mathrm{P}<0.05$ was considered to indicate a statistically significant difference.

\section{Results}

Clinical characteristics. The clinical characteristics of the study population are shown in Table I. MetS prevalence was $45.73 \%(n=75)$. OxS was observed in $42.68 \%$ of the participants, including $64 \%, \mathrm{n}=48 / 75$ women with MetS and $24.7 \%, \mathrm{n}=22 / 89$ women without it $(\mathrm{P}<0.001)$. Mean TAC in women with and without MetS was $1.505 \pm 0.0453 \mathrm{mmol} / \mathrm{l}$ and $1.304 \pm 0.045 \mathrm{mmol} / \mathrm{l}$, respectively $(\mathrm{P}<0.001)$.

Multiple linear regression analysis and number of MetS components. To evaluate the association between MetS components with MDA and TAC levels, a multiple linear regression analysis was performed (Table II). MDA and TAC levels showed a significant association with WC, TG, HDL-C and FBG independently. TG was the factor most strongly associated with MDA and TAC, followed by HDL-C according to the standardized B. Table III shows the mean \pm standard deviation MDA and TAC according to the number of MetS components. MDA increased, but TAC decreased with an increase in the number of MetS components $(\mathrm{P}<0.001)$.

Independent effects of MetS and aging on the MDA and TAC levels. Independent effects of MetS and aging on the MDA and TAC levels are shown in Table IV. The results revealed that although the MetS and age were independent risk factors for OxS and the antioxidant activity reduction, the predictive power of the MetS was much more than that of age (standardized B 0.573 for MetS and 0.376 for age).

MDA and TAC quartiles. The characteristics of the participants according to the MDA and TAC quartiles are shown in Table V. The mean age, WC, TG and FBG increased and HDL-C decreased with MDA from the lowest to the highest quartile. By contrast, the mean age, WC, TG, and FBG decreased and HDL-C increased significantly with TAC from the lowest to the highest quartile.

\section{Discussion}

Previous findings have shown an increased level of OxS among frail, institutionalized elderly individuals which may consequently result in an accelerated aging process, and elevated incidence of oxidative diseases including cancers, CVD, or dementia (18). Similar findings of increased oxidant parameters and a decrease in antioxidants in patients with MetS have been reported in previous studies (19-21). However, there is a lack of information on the interaction between MetS and aging over the OxS status among elderly women. Previous studies in this area were predominantly conducted on individuals with multi-metabolic disorders (such as diabetes, hypertension, or hyperlipidemia) $(8,14,16,20)$. By contrast, our study population sample had no metabolic or specific established diseases. Therefore, we simultaneously addressed the association of stress oxidation with MetS and senescence in an indepently living healthy elderly population. 
Table IV. Correlation of the MetS and age with MDA and TAC in elderly women $(n=164)$.

\begin{tabular}{|c|c|c|c|c|c|c|}
\hline \multirow[b]{2}{*}{ Parameters } & \multicolumn{3}{|c|}{ MDA } & \multicolumn{3}{|c|}{ TAC } \\
\hline & B (SE) & $\beta$ & P-value & B (SE) & $\beta$ & P-value \\
\hline MetS (yes vs. no) & 0.865 & 0.573 & $<0.001$ & -0.078 & -0.005 & $<0.001$ \\
\hline Age $\geq 65$ years (vs. age $60-65$ ) & 0.489 & 0.376 & $<0.001$ & -0.057 & -0.003 & $<0.001$ \\
\hline
\end{tabular}

MetS, metabolic syndrome; MDA, malondialdehyde; TAC, total antioxidant capacity.

Table V. Characteristics of participants according to the quartiles of MDA and TAC $(n=164)$.

\begin{tabular}{lccccr}
\hline & Q1 & Q2 & Q3 & Q4 & \\
Characteristics & $(2.56-4.048)$ & $(4.049-4.83)$ & $(4.84-5.67)$ & $(5.68-9.32)$ & P-value \\
\hline MDA & & & & \\
Age & $62.50 \pm 3.14$ & $63.95 \pm 2.89$ & $65.41 \pm 3.28$ & $66.20 \pm 2.83$ & $<0.01$ \\
WC & $89.47 \pm 6.49$ & $93.29 \pm 9.81$ & $94.05 \pm 8.45$ & $96.94 \pm 8.57$ & $<0.01$ \\
SBP & $127.05 \pm 5.32$ & $126.47 \pm 3.42$ & $127.64 \pm 4.71$ & $128.52 \pm 4.24$ & 0.58 \\
DBP & $79.11 \pm 5.07$ & $82.17 \pm 4.15$ & $79.31 \pm 5.37$ & $82.35 \pm 7.52$ & 0.26 \\
TG & $205.82 \pm 22.61$ & $210.17 \pm 24.56$ & $213.23 \pm 24.86$ & $219.64 \pm 28.69$ & $<0.001$ \\
HDL-C & $53.14 \pm 2.56$ & $50.42 \pm 3.25$ & $48.25 \pm 2.86$ & $46.58 \pm 2.38$ & $<0.001$ \\
FBG & $96.25 \pm 8.40$ & $101.48 \pm 8.36$ & $103.94 \pm 10.63$ & $104.70 \pm 8.49$ & $<0.001$ \\
\hline & & & & \\
Characteristics & $(0.879-1.169)$ & $(1.170-1.289)$ & $(1.290-1.414)$ & $(1.415-2.111)$ & P-value \\
\hline TAC & & & & & \\
Age & & & & & \\
WC & $65.38 \pm 2.76$ & $65.25 \pm 2.28$ & $63.31 \pm 2.89$ & $63.28 \pm 3.14$ & $<0.01$ \\
SBP & $97.41 \pm 10.58$ & $95.70 \pm 8.45$ & $95.35 \pm 9.80$ & $90.29 \pm 8.92$ & $<0.01$ \\
DBP & $127.47 \pm 4.59$ & $126.76 \pm 4.65$ & $127.05 \pm 3.56$ & $129.41 \pm 4.63$ & 0.19 \\
TG & $81.17 \pm 5.16$ & $80 \pm 6.12$ & $80.45 \pm 6.28$ & $80.29 \pm 5.72$ & 0.94 \\
HDL-C & $217.58 \pm 19.46$ & $216 \pm 15.69$ & $215.94 \pm 18.40$ & $199.35 \pm 26.40$ & $<0.001$ \\
FBG & $48.32 \pm 2.95$ & $52.33 \pm 3.24$ & $51.88 \pm 2.22$ & $52.50 \pm 1.06$ & $<0.01$ \\
& $108.41 \pm 10.13$ & $101.29 \pm 10.88$ & $103.70 \pm 10.25$ & $98.23 \pm 10.92$ & $<0.01$ \\
\hline
\end{tabular}

Values are mean \pm standard deviation. WC, waist circumference; SBP, systolic blood pressure; DBP, diastolic blood pressure; TG, triglyceride; HDL-C, high-density lipoprotein cholesterol; FBG, fasting blood glucose; MDA, malondialdehyde; TAC, total antioxidant capacity.

In the present study, MDA and TAC levels were found to be significantly higher and TAC levels decreased in the presence of MetS. Among the MetS components, TG, HDL-C, WC and FBG were the strongest predictors on the levels of MDA and TAC independently or in combination, respectively. This finding is consistent with recent findings (3), whereby the individual components of MetS were associated with a reduced TAC and increased MDA. However, Abdilla et al reported that the contribution of components of MetS towards $\mathrm{OxS}$ in MetS is minimal and the OxS observed is mainly due to hypertension (22). In the present study, hypertension was not recognized as an important predictor in determining the level of TAC and MDA. This finding is in good agreement with the finding of Francisqueti et al, which indicated that the TAC is not associated with blood pressure less or greater than 130/85 mm/hg (4). Lohr et al also confirmed this finding. Authors of that study indicated that the blood pressure was not associated with the oxidatively damaged DNA levels among individuals with MetS (23). By contrast, Najarzadeh et al (24) and Bitla et al (19) showed a significant correlation among these variables.

One reason behind this variation may be due to the fact that our study samples were in a pre-hypertension status and had no significant difference compared to the control group in terms of blood pressure. Moreover, the findings in the association between MDA and TAC and MetS components in various metabolic conditions, have been shown to vary. A comparison of TAC among physically active ex-athletes and sedentary ex-athletes has shown no difference, while TG and TC were significantly elevated in sedentary ex-athletes (25). 
A study conducted on psoriasis patients demonstrated a lower level of TAC in addition to elevated TG and TC compared to the control groups (26).

Moreover, the study conducted by Bitla et al demonstrated that FBG, TG and WC levels were positively correlated with MDA levels but negatively associated with FRAP among individuals with MetS. By contrast, the mean FBG, TG and WC were indicative of diabetes, hypertriglyceridemia and abdominal obesity in all the participants of that study (19). Similarities in the results of our study with the study of Bitla et al indicate that the MetS can increase the risk of OxS, even in non-diabetic and non-hyperlipidemic cases (19).

Our findings also showed a positive correlation between increasing age with $\mathrm{OxS}$. Age increase especially in women can elevate the risk of $\mathrm{OxS}(1,5,23)$. Previous data also revealed higher protein oxidation level in women compared to men. Additionally, postmenopausal women demonstrated higher protein oxidation level compared to younger premenopausal women (6). The study results of Dalvand et al are consistent with this finding (27). However, the correlation between aging and lipoperoxide production levels changes has not been previously reported in other studies $(1,6)$. Diversity of lifestyle-related factors and the participants' age at the time of enrolment for the studies may have impacted the result and may be the reason behind the discrepancies of study results. For instance, participants' lifestyles varied from institutionalized, hospitalized, disabled or independent. Moreover, the subjects are considered as older at $60,70-80$, or 80 years which varies depending on each study.

Measurements in older subjects in our study demonstrated lower levels of plasma total FRAP; the decrease was age-related. These results strongly suggest an age-related risk of OxS in elderly subjects. The accumulation of OxS is one of the most significant elements linked to aging and age-related chronic diseases. Aging has been demonstrated to result in a loss of fat mass and OxS accumulation in adipose tissue (6). The lifetime accumulation of ROS potentially results in direct cell damage. In addition to the accumulation of oxidative damage throughout a life span, aging is directly linked to an impaired antioxidant defense mechanism. An impaired antioxidant defense may result in elevation of ROS levels and impaired degradation, which eventually would directly and indirectly damage the organs. Therefore, $\mathrm{OxS}$ in aging, besides being directly linked to organ damage, would exacerbate the risk factors of vascular damage including $\operatorname{MetS}(3,18)$.

The aging process is altered when metabolic and CVD are present and the risk of disease increases with age. Many predisposing conditions, which increase in prevalence during aging, such as obesity, IR, inflammation, changes in the activity of the hypothalamus-hypophysis axis, stress and hypertension, also contribute to increasing the prevalence of MetS. Aging, the development of IR and CVD seem to be accelerated in the MetS (28).

In this study, the comparison of the MetS predictive effect and age on OxS showed that MetS is a much stronger predictor than age. Multivariable analysis revealed that both MetS and age above 65 can aggravate the risk of CVD through the development of OxS. The studies of Bonomini et al (29) and Veronica and Esther (30) also showed that OxS in elderly individuals with MetS, becomes exacerbated with age. However, the role of genetic and environmental factors and diet in causing the oxidation remain to be determined.

Based on this finding, some researchers have reported the beneficial effect of antioxidant vitamin supplementation on decreasing CVD risk in MetS patients (15) while others showed no effect $(31,32)$. However, the majority of those studies have not monitored the improvement in antioxidant status with antioxidant therapy. Therefore, antioxidant therapy monitored with the use of FRAP assay along with appropriate lifestyle modification to decrease abdominal obesity and modification lipid may help in blocking the effects of OxS and improve the antioxidant defense and thus the prevention of CVD complications and healthy aging.

There were some limitations to this study. First, that this was a cross-sectional study is not conducive to determining a causal relationship between MetS and aging with OxS markers. Second, this study was conducted among elderly women and the association between OxS and the variables of MetS may be different in elderly men. In order to confirm the mechanism underlying the documented relationships of this study, prospective designed studies are required.

Our findings highlighted that although aging increased the OxS status and reduced the antioxidant defense, the impact of MetS on these disorders is far greater than the effect of age. Thus, their cumulative effects can lead to an increased CVD risk among elderly individuals. Therefore, the early diagnosis of MetS and awareness of the OxS status in elderly individuals can prevent the adverse impact of MetS on OxS and reduce the effects of a slow and progressive reduction of antioxidant activity due to aging. Further exploration of this research for the role of OxS in the MetS and the related accelerated senescence may contribute to the development of further therapies.

\section{Acknowledgements}

The present study was supported by a research grant from Babol University of Medical Sciences, Iran. We would like to thank all of the participants in the study as well as the staff at the rural health clinics of Babol, Iran, for their good cooperation in this study.

\section{References}

1. Manda-Handzlik A and Demkow U: Neutrophils: The role of oxidative and nitrosative stress in health and disease. Adv Exp Med Biol 857: 51-60, 2015.

2. Ho E, Karimi Galougahi K, Liu CC, Bhindi R and Figtree GA: Biological markers of oxidative stress: Applications to cardiovascular research and practice. Redox Biol 1: 483-491, 2013.

3. Jansen EH and Ruskovska T: Comparative analysis of serum (anti)oxidative status parameters in healthy persons. Int J Mol Sci 14: 6106-6115, 2013

4. Francisqueti FV, Chiaverini LC, Santos KC, Minatel IO, Ronchi CB, Ferron AJ, Ferreira AL and Correa CR: The role of oxidative stress on the pathophysiology of metabolic syndrome. Rev Assoc Med Bras (1992) 63: 85-91, 2017.

5. Tiganis T: Reactive oxygen species and insulin resistance: The good, the bad and the ugly. Trends Pharmacol Sci 32: 82-89, 2011.

6. Romano AD, Serviddio G, de Matthaeis A, Bellanti F and Vendemiale G: Oxidative stress and aging. J Nephrol 23 (Suppl 15): S29-S36, 2010.

7. Ziaei S, Sayahi M and Faghihzadeh S: Relationship between reproductive aging, body composition, hormonal status and metabolic syndrome in postmenopausal women. Climacteric 14: 649-653, 2011. 
8. Ganjifrockwala F, Joseph J and George G: Decreased total antioxidant levels and increased oxidative stress in South African type 2 diabetes mellitus patients. JEMDSA 22: 21-25, 2017

9. Delavar R, Mogharnasi M and Khoobkhahi N: The effects of combined training on oxidative stress and antioxidant defense indicators. Int J Basic Sci Med 2: V29-V32, 2017.

10. Inglés M, Gambini J, Carnicero JA, García-García FJ, Rodríguez-Mañas L, Olaso-González G, Dromant M, Borrás C and Viña J: Oxidative stress is related to frailty, not to age or sex, in a geriatric population: Lipid and protein oxidation as biomarkers of frailty. J Am Geriatr Soc 62: 1324-1328, 2014.

11. Hajian-Tilaki K: Metabolic syndrome and its associated risk factors in Iranian adults: Caspian J Intern Med 6: 51-61, 2015.

12. Hajian-Tilaki K, Heidari B, Firouzjahi A, Bagherzadeh M, Hajian-Tilaki A and Halalkhor S: Prevalence of metabolic syndrome and the association with socio-demographic characteristics and physical activity in urban population of Iranian adults: A population-based study. Diabetes Metab Syndr 8 : 170-176, 2014.

13. Adib-Hajbaghery $M$ and Aghahoseini S: The evaluation of disability and its related factors among the elderly population in Kashan, Iran. BMC Public Health 7: 261, 2007.

14. Taslim S and Tai ES: The relevance of the metabolic syndrome. Ann Acad Med Singapore 38: 29-35, 2009.

15. Ruiz-Ramos M, Vargas LA, Fortoul Van der Goes TI, CervantesSandoval A and Mendoza-Nunez VM: Supplementation of ascorbic acid and alpha-tocopherol is useful to preventing bone loss linked to oxidative stress in elderly. J Nutr Health Aging 14 467-472, 2010

16. Cerniauskiene LR, Luksiene DI, Tamosiūnas A, Reklaitiene R and Margeviciene L: Association of metabolic syndrome and oxidative stress with ischemic heart disease in middle-aged persons. Medicina (Kaunas) 44: 392-399, 2008 (In Lithuanian).

17. Benzie IF and Strain JJ: The ferric reducing ability of plasma (FRAP) as a measure of 'antioxidant power': The FRAP assay. Anal Biochem 239: 70-76, 1996.

18. Maugeri D, Santangelo A, Bonanno MR, Testai M, Abbate S, Lo Giudice F, Mamazza C, Pugllsi N and Panebianco P: Oxidative stress and aging: Studies on an east-sicilian, ultraoctagenarian population living in institutes or at home. Arch Gerontol Geriatr Suppl 38: 271-277, 2004.

19. Bitla AR, Kumari NM, Reddy NS, Nagaraju KV, Sachan A, Kumar VP, Suchitra MM and Srinivasa Rao PVLN: Antioxidant status in patients with metabolic syndrome as measured by ferric reducing ability of plasma (FRAP) assay. J Clin Sci Res 3: 114-120, 2012

20. Carrier A: Metabolic syndrome and oxidative stress: A complex relationship. Antioxidants \& Redox Signaling 26: 429-431, 2017.
21. Picu A, Petcu L, Stefan S, Mitu M, Lixandru D, Ionescu-Tîrgovişte C, Pîrcălăbioru GG, Ciulu-Costinescu F, Bubulica MV and Chifiriuc MC: Markers of oxidative stress and antioxidant defense in romanian patients with Type 2 diabetes mellitus and obesity. Molecules 22: E714, 2017.

22. Abdilla N, Tormo MC, Fabia MJ, Chaves FJ, Saez G and Redon J: Impact of the components of metabolic syndrome on oxidative stress and enzymatic antioxidant activity in essential hypertension. J Hum Hypertens 21: 68-75, 2007.

23. Lohr M, Jensen A, Eriksen L, Grønbæk M, Loft S and Møller P: Age and metabolic risk factors associated with oxidatively damaged DNA in human peripheral blood mononuclear cells. Oncotarget 6: 2641-2653, 2015.

24. Najarzadeh A,Mozaffari-KhosraviH,Mahdavi M,FallahzadehH, Zavar-Reza J and Shahmoradi H: Antioxidant status in patients with metabolic syndrome as measured by the stable free radical diphenylpicrylhydrazyl assay. IJDO 4: 161-166, 2012.

25. Pihl E, Zilmer K, Kullisaar T, Kairane C, Pulges A and Zilmer M: High-sensitive C-reactive protein level and oxidative stress-related status in former athletes in relation to traditional cardiovascular risk factors. Atherosclerosis 171: 321-326, 2003.

26. Vanizor Kural B, Orem A, Cimsit G, Yandi YE and Calapoglu M: Evaluation of the atherogenic tendency of lipids and lipoprotein content and their relationships with oxidant-antioxidant system in patients with psoriasis. Clin Chim Acta 328: 971-982, 2003.

27. Dalvand S, Niksima SH, Meshkani R, Ghanei Gheshlagh R, Sadegh-Nejadi S, Kooti W, Parizad N, Zahednezhad H and Afrisham R: Prevalence of metabolic syndrome among iranian population: A systematic review and meta-analysis. Iran J Public Health 46: 456-467, 2017.

28. Guarner-Lans V, Rubio-Ruiz ME, Pérez-Torres I and Baños de MacCarthy G: Relation of aging and sex hormones to metabolic syndrome and cardiovascular disease. Exp Gerontol 46: 517-523, 2011.

29. Bonomini F, Rodella LF and Rezzani R: Metabolic syndrome, aging and involvement of oxidative stress. Aging Dis 6: 109-120, 2015 .

30. Guarner-Lans V and Rubio-Ruiz ME: Aging, metabolic syndrome and the heart. Aging Dis 3: 269-279, 2012.

31. Myung SK, Ju W, Cho B, Oh SW, Park SM, Koo BK and Park BJ: Korean meta-analysis study group: Efficacy of vitamin and antioxidant supplements in prevention of cardiovascular disease: Systematic review and meta-analysis of randomised controlled trials. BMJ 346: f10, 2013.

32. Ye Y, Li J and Yuan Z: Effect of antioxidant vitamin supplementation on cardiovascular outcomes: A meta-analysis of randomized controlled tri https. PloS One 8: e56803, 2012. 